IPSI Revista de Investigación en Psicología.

1998, Vol. 1, No 1.pp. 31-58

\title{
PERSONALIDAD Y DESEMPLEO *
}

\section{Dora Frisancho Vera**}

\begin{abstract}
Anal Fairlie Altez***
Se aplica el Rorschach a un grupo de despedidos y a otro de trabajadores en ejercicio de la Municipalidad de Lima. Las diferencias inciden en incremento de respuestas de detalle pequeño y color acromatico además de disminución de respuestas populares, que subrayan una restricción del campo perceptual y del sentido común en los despedidos.

Palabras clave: Desempleo, despido, personalidad, prueba de Rorschach, sistema comprehensivo de Exner.

It is applied the Rorschach to a group of bidden farewell and to other of workers in exercise of the Municipality of Lima, Peru. The differences impact in answers increase little detail and acromatic color so decrease of popular answers, that underline a restriction of the perceptual field and of the common sense in the bidden farewell.
\end{abstract}

Key words: Employ, bid den farewell, personality, Rorschach test, Exner's comprehensive system

El tema del desempleo interesa no solamente a las ciencias ligadas con la producción sino a todas las ciencias y a todo el conocimiento en tanto afecta la actividad esencial del hombre que es el trabajo, la razón de ser del hombre de nuestro tiempo. Quedarse sin empleo altera la ubicación del individuo en la sociedad y en la familia y altera también, en no menor grado, el equilibrio personal. A la psicología le ha preocupado sobretodo el impacto que ocasiona en la personalidad y de ello tenemos referencias sobre estudios efectuados en otras partes. En nuestro país el desempleo ha sido tocado sólo desde el interés de la economía, y lo que se ha dicho sobre su repercusión en las personas no pasa de comentarios y puntos de vista

* Colaboradores: Efraín Flores, Emma Infante y Elena Peña.

** Email d280016@unmsm.edu.pe.

*** Email d280013@unmsm.edu.pe. 
sin estudios especiales centrados en la psicología de ese fenómeno. Esta vez vamos al encuentro de una de las formas menos deseables del desempleo, que es la que se produce por despido laboral, y hemos tratado de ver sus efectos en los trabajadores de nuestro medio escudriñando las áreas psíquicas que le son más sensibles y los cambios registrables en los rasgos de personalidad.

El resultado es modesto, pero nos satisface. Por un lado confirma las repercusiones negativas descritas por otros autores y que son ya de conocimiento general y por otro destaca algunos rasgos de personalidad que ayudan a comprender la experiencia cercana del trabajador despedido, uno de los cuales tiene que ver con el sentido común de percibir las cosas que en los trabajadores del estudio se restringe y complica en sentido incapacitante.

Se trata de un primer estudio que lo hemos circunscrito a un grupo de trabajadores de un sólo sindicato, comparando lo que acontece en la personalidad de los que han sido despedidos respecto de los que siguen trabajando. Esperamos contar con circunstancias favorables para ampliar estas primeras observaciones y verificar sus alcances.

\section{EL DESEMPLEO}

\section{Algunos Criterios}

El fenómeno de la desocupación o desempleo ha estado siempre ligado a los procesos productivos de los diferentes países. En los llamados países socialistas, la desocupación parecía haberse reducido considerablemente, pero ese logro casi se ha desvanecido con la reciente disolución de la U.R.S.S. y se espera que no afecte en la misma medida a otros países de esa órbita como China y Cuba En occidente se siente actualmente la preocupación de los gobiernos y de los organismos competentes por las crecientes tasas de desempleo. Mientras la situación laboral se aproximaba al grado de pleno empleo esa preocupación era mínima y hasta se consideraba normal o justificado un cierto porcentaje de desocupados; pero en las últimas dos décadas el fenómeno ha crecido en dimensiones alarmantes, tanto que la propia OMS lo ha calificado como "la principal catástrofe epidemiológica" de nuestro tiempo. 
Entre lo que sucedía antes y lo que se ve ahora, hay una diferencia que castiga a la sociedad entera. Es notoria la ausencia de esos grandes desplazamientos de trabajadores saliendo de las factorías al toque de la sirena y los movimientos del proletariado en sindicatos y partidos políticos y, de vez en cuando o, más espacialmente, se dejan oir los grupos de cesados reivindicando los derechos perdidos. Es una situación real de que ya no hay empleo como antes y esto merece la explicación de los más diversos autores y de los más diversos puntos de vista, no siendo pocas las veces que esta diversidad afecta a un mismo autor. No corresponde aquí -ni tendríamos posibilidades de hacerlo- intentar una relación o un repaso de los distintos criterios, sino sólo mencionar algunos que dan fe de las explicaciones más recurrentes y de los que circulan en los medios intelectuales.

En una exposición coherente, Nelson Manrique (1997) nos dice que en el trabajo como en varios otros órdenes de la realidad, se viene dando un radical proceso de desmasificación mediante el cual pierde vigencia el trabajador-masa, de esos que se contaban por millones y constituían una importante fuerza política, a raíz de los cambios originados por la "tercera revolución industrial" y del mayor valor otorgado a la información y al conocimiento. Estos cambios han hecho de las grandes empresas y de las "economías de escala" modos de producción innecesarios y costosos.

La escala óptima de producción ya no es más la de la gran empresa: engorrosa, lenta y complicada de manejar, sino la de la pequeña y mediana empresa, sencillas en su estructura, con una menor cantidad de unidades que coordinar y con la tecnología de las redes informáticas para facilitar los enlaces. La estructura empresarial se "achata" reduciendo la cantidad de escalones funcionales de comando. Esta menor distancia entre la dirección y los escalones inferiores de la empresa no responde pues tanto a una "democratización" de las estructuras empresariales, cuanto a la necesidad de agilizar la conducción de las compañías y reducir los costos administrativos, lo que se ha hecho posible gracias al empleo de las nuevas tecnologías informáticas y telemáticas. Pero esta forma de producción supone reintegrar tareas que antes estaban separadas por la división social del trabajo imperante, y esto exige, un nuevo tipo de trabajador capaz de realizar actividades, diversas, a diferencia del trabajador, que sólo realizaba una tarea simple y repetitiva. Las consecuencias de estos cambios son drásticas. 
Vivimos, en primer lugar, la decadencia del proletariado clásico, ese que laboraba en grandes fábricas, donde miles de trabajadores compartían no sólo un trabajo semejante sino problemas similares y un estilo de vida característicos. Así, entraron en crisis el sindicalismo, los partidos obreros y el proyecto político que los aglutinaba. La búsqueda de la justicia y la equidad entre los hombres dejó de correr por el carril de la producción masiva. (p.55).

Reducida la mediación administrativa y la división social del trabajo, se reduce concomitantemente el número de puestos de trabajo y crece desmesuradamente el desempleo.

Contra las previsiones de los sesenta cuando se consideraba que el pleno empleo era el resultado del crecimiento de la producción, y las del setenta, que concluyeron que el empleo no dependía necesariamente del crecimiento (ni viceversa), en los noventa se impone la conclusión de que la desocupación es la condición del crecimiento. Aunque entre 1960 y 1987 el producto nacional bruto ha crecido en Gran Bretaña 83\%, en Alemania $122 \%$ y en Francia $168 \%$, hoy la desocupación es mayor a la de 1960 en los tres países (y citando a F. Mires): El proyecto de disminuir las tasas de ocupación para "crecer" ya se había probado exitoso en algunos países latinoamericanos. Reaganismo y Thatcherismo, fueron las formas políticas que asumió en los países más industrializados. Hoy alcanza su pleno apogeo en el Este de Europa. (ob. cit., p.127).

Otras hipótesis localizan la fuente del desempleo en las necesidades de ajuste de la balanza de pagos internacional y las estrategias políticas de respuesta, resultando ser tina opción de política económica:

La esencia del problema de ajuste es que cada política implica costos económicos. Por ejemplo, para Corregir su déficit externo, un país puede tener que deflactar su economía y aceptar desempleo doméstico. Un país con superávit puede eliminar su superávit indeseado teniendo que aceptar inflación. (Chacholiades, 1992, p. 359).

y esto deriva de las condiciones de oferta y demanda que afectan al país, de modo que si por ejemplo hay exceso de oferta, los productores tendrán que disminuir la producción con el consiguiente decremento del ingreso nacional y la imposibilidad de mantener el 
equilibrio externo. Refiriéndose al país del norte, el mismo Chacholiades dice que:

... si no puede reducirse la tasa de salario monetario, entonces los productores norteamericanos simplemente tendrán que recortar su producción de exportables, puesto que no puede esperarse que acumulen inventarios de manera indefinida. Así, los productores comenzarán a despedir trabajadores y la tasa de desempleo de los Estados Unidos comenzará a aumentar, a medida que la producción y el ingreso nacional caen (lbid., p.365).

Viviane Forrester (1997) descarna el problema del desempleo, sobre todo en lo que significa para quienes lo padecen. Afirma enfáticamente que el mundo ha cambiado y que nos resistimos a ver el cambio. Lo evidente es que ya no hay trabajo o es muy escaso en relación a los millones de "conciencias torturadas y negadas una por una". Sostiene que el desempleo actual no es objeto de una marginación transitoria, ocasional o reducida sólo a algunos sectores, sino que es ya lo general y lo vigente. Y el desempleado:

... está atrapado por una implosión general, un fenómeno comparable con esos maremotos, huracanes o tomados que no respetan a nadie y a quien nadie puede resistir. Es víctima de una lógica planetaria que supone la supresión de lo que se llama trabajo, es decir, de los puestos de trabajo ( p. 13)

Insiste en que seguimos aferrados al recuerdo del trabajo sin querer admitir que ya no existe y, en un absurdo difícil de comprender, hacemos como que lo social y lo económico están regidos por las transacciones realizadas a partir del trabajo. Nos movemos en ese desfase entre lo que fue y lo que ya no es. La organización de lo social ha cambiado radicalmente. El trabajo que permite la vida digna sólo alcanza para algunos, que resultan privilegiados, y entonces se pregunta, "en una pregunta esencial jamás formulada" si es necesario merecer el derecho de vivir.

Una ínfima minoría, provista de poderes excepcionales, propiedades y derechos considerados naturales, posee de oficio ese derecho. En cambio, el resto de la humanidad, para "merecer" el derecho de vivir, debe 


\section{Dora Frisancho / Anal Fairlie}

demostrar que es "útil" para la sociedad, es decir, para aquello que la rige y la domina, la. economía de mercado. Para ella, "útil", significa siempre "rentable", es decir, que le dé ganancia a las ganancias. En una palabra, significa "empleable" ("explotable" seria de mal gusto) (lb. p.15).

Es como si el trabajo hubiera prescrito, pues los puestos que le dan acceso están permanentemente cerrados, y han prescrito, dice, debido a la ineficiencia general, el interés de algunos o el curso de la historia, "todo colocado bajo el signo de la fatalidad". En esa situación, y en otra de sus frecuentes interrogantes se pregunta si es normal o lógico imponer aquello que falta por completo, o si es legal imponer como condición necesaria para la supervivencia lo que no existe. Y por este camino de reflexión se ubica en el pellejo de los detentadores del poder económico que deben estar de plácemes al ver a sus pies a esos agitadores que ayer nomás bullían reclamando, reivindicando y combatiendo: "que placer verlos implorar por aquello que hasta ayer denostaban y hoy anhelan con fervor". Y no menos placer deben sentir por el cuidado que ponen los que en posesión todavía de salario temen perder su privilegio por no encontrarse a la cola de los hundidos en la miseria. Y de esta manera, remata Forrester, el sistema social nos lleva;

... de la explotación a la exclusión, de ésta a la eliminación e incluso a desastrosas explotaciones aún desconocidas: ¿es ésta una hipótesis inconcebible? Sabemos por experiencia que la barbarie, siempre latente, se conjuga de maravillas con la mansedumbre de esas mayorías que saben incorporar el horror a la frivolidad ambiente (lb. p.20).

El problema del desempleo tenía que haber ocupado también a los clásicos de la economía como Keynes, uno de los autores más comentados de este siglo, que ya desde 1936 destacaba la necesidad de estudiarlo como asunto de importancia y respecto del cual afirmaba que:

No hay nada que garantice el pleno empleo, al contrario, el sistema económico funciona de tal manera que lo normal es que exista el desempleo (involuntario). No hay tal funcionamiento automático del Sistema económico de mercado que asegure su estabilidad y el pleno empleo (Verdera, 1983, p. 34). 
Clásicos y modernos, extranjeros y nacionales, los pronunciamientos se suceden interminablemente y a todos los asemeja esa incertidumbre keynesiana que parece tener mayor asidero cuando se trata de los llamados países subdesarrollados.

En nuestro país todos los indicadores económicos de los últimos 40 años corresponden a la hoja clínica de un enfermo, con curvas y líneas que suben o bajan violentamente. Si se compara la evolución de estas variables con la registrada en cualquier país desarrollado se ve claramente la diferencia (Boloña. 1993, p.1).

Y páginas después, este último autor nacional citando a la revista Perú Económico, denomina a ese panorama "auténtica radiografía de un desastre", y se alinea luego con quienes sostienen la necesidad de reducción de los puestos de trabajo: "No se puede reducir la inflación sin desempleo y recesión" (p. 64).

Y tampoco es infrecuente encontrar, también entre los nuestros, quienes subrayan la intencionalidad política en el manejo económico que, acatando designios de grupo o interés, promueven entre otras cosas desbarajustes en el trabajo:

En evidente relación con esta crisis, ha surgido en el Perú un proyecto liberal, fondo monetarista para el lenguaje económico y político, amenazante para el conjunto de los trabajadores a quienes se les pide escrito en 1990- dos o tres años más de sacrificio, en un momento en el que el salario casi ha desaparecido. (Bernedo, 1990. p.62).

y casi enseguida va a añadir: "Más de la mitad de desempleados son trabajadores (cesantes) que han perdido el empleo precisamente por estar desprotegidos o porque no se cumple la ley". (lb. p.73).

Aún cuando en la práctica económica y en el rollo teórico se admite el desempleo como necesidad, sin embargo, es universal el pronunciamiento en su contra, así de autores cuanto de funcionarios. El presidente de la Sociedad de Estudios de América Latina, peruano, sostuvo que: 
El desempleo en el país está más relacionado con el ajuste económico que con la explosión demográfica, más conocida como "baby boom", y que la responsabilidad de combatirla es de la sociedad en su conjunto porque la producción depende de los peruanos en conjunto".("El Comercio", 02-1197).

Nadie está seguro de si los esfuerzos por combatirla son los más apropiados, pero los gobiernos como el nuestro oponen como respuesta los llamados programas de ayuda social que suelen ser esfuerzos extremadamente limitados.

No hay Programa de Apoyo Social capaz de neutralizar el perjuicio acusado por un tipo de ajuste cuyo éxito depende positivamente de la magnitud del daño causado a la economía y a los trabajadores. (Iguiñiz, en Bemedo,1990, p. 369)

También aparecen, naturalmente, pronunciamientos y movilizaciones, y han comenzado a surgir organizaciones de desocupados, como la red europea ENU (European Network of the Unemployed ("Brecha", 20-0697), que por ahora alcanzan limitada repercusión.

\section{Consecuencias del desempleo}

Sobre los efectos del desempleo hay versiones que proceden de las más diversas fuentes, así de profanos como de especialistas, y tienen expresión en artículos periodísticos, revistas y libros especializados. De todas ellas tienen interés para nosotros las que se vinculan de una u otra manera a la psicología. Tampoco aquí vamos a pretender hacer un recuento de todo lo que se ha tratado al respecto, sino presentar tan sólo una referencia general a la índole de los estudios suficientes para dar contexto al punto específico que nos ocupa.

Los españoles Peiró y Prieto ofrecen un excelente resumen de lo que se ha venido ventilando en este campo. Dos son los temas que han absorbido la mayor dedicación: el que concierne a los antecedentes o causas del desempleo y el que se refiere a su impacto a nivel individual y familiar. El primero abarca estudios que no sólo acuden a las "variables macro" 
de orden socioeconómico como la razón entre oferta y demanda de empleo, el papel de la recesión o la existencia de políticas de fomento de empleo, sobre las que el individuo tiene escaso control sino también a variables demográficas del desempleado como la edad, el sexo, el nivel de instrucción que hacen accesibles o alejan las posibilidades de empleo, y aun variables o características psicológicas que hacen al sujeto más empleable o lo toman más o menos activo en la búsqueda de empleo. Nuestra preocupación reside en el tema del impacto del desempleo en el trabajador.

Respecto a cuál es la repercusión del desempleo en la persona, los estudios que resumen los autores españoles citados, unas veces aluden al efecto diferencial que ocasiona según el afectado sea adulto o joven, incidiendo en su mayoría que el mayor sufrimiento lo cargan los adultos entre 30 y 45 años y en los casados más que en los solteros. Otras veces la referencia es al nivel de análisis donde hay un predominio de los datos agregados de medida y su correlación con las tasas de salud mental que suelen concluir, por ejemplo, en la correspondencia entre los cambios en el índice de desempleo con los cambios en índices de mortalidad, suicidio y solicitudes de los servicios de salud. Otras veces, los estudios descansan en el método o enfoque que puede ser cualitativo -mediante el estudio de casos o la observación participante, entre otros- o cuantitativo con uso de técnicas de medida validadas, procedimientos de muestreo y "sofisticados análisis estadísticos" ( Peiró y Prieto, 1996, p. 237). En el concepto de autores como Fryer ambos muestran continuidad y similitud en sus resultados. Paralelamente se cuentan estudios que se distinguen por el diseño de investigación, siendo unos transversales y otros longitudinales con resultados que tienden a complementarse mutuamente. Por último, otros estudios se diferencian por el tipo de consecuencias que pueden ser físicas o somáticas con registros como: dolores de cabeza, pérdida de apetito, disneas, mareos, insomnio e hipertensión, y consecuencias psicosociales centradas en indicadores de salud mental y bienestar psicológico.

Nuestro estudio encaja con el último criterio mencionado, que se refiere directamente a estados mentales y contenidos de personalidad. En este sentido, cabe ahora hacer referencia a estudios encaminados a determinar 
consecuencias psicosociales que van a servimos de punto de comparación o de partida a los efectos de nuestro propósito de estudio.

En la mayoría de consultas, las versiones sobre consecuencias del desempleo en las personas son expuestas en forma general y escueta. Por ejemplo, en el resumen de los citados autores españoles y en relación a una serie de estudios longitudinales, se lee:

En general, los resultados de estas investigaciones confirman los obtenidos en los estudios transversales en el sentido de que las personas desempleadas tienen un menor bienestar psicológico que las empleadas. (ob, cit., p. 239).

Hacen mención luego a Winefield y colaboradores que siguen longitudinalmente a 554 jóvenes desde que eran estudiantes y no mostraban entre sí diferencias en bienestar psicológico; pero que luego constituyen cuatro grupos laborales, a saber: Desempleados, empleados satisfechos con su trabajo, empleados no satisfechos con su trabajo y estudiantes, que es cuando aparecen diferencias significativas en sentimientos depresivos, estado de ánimo negativo e indefensión. Refieren también que se encuentran resultados contradictorios entre algunos estudios, como que unos afirman que el desempleo conlleva deterioro de la salud mental y otros que si bien el empleo se asocia a mejora en el bienestar psicológico, el desempleo no conduce a deterioro de la salud mental, no faltando quienes aseguran que el paso de los estudios al trabajo, sea como empleados o desempleados, conlleva un "ligero aumento" del bienestar psicológico. Estos cambios invitan a Neff a decir: "No es de extrañar, por lo tanto, que los hallazgos contradictorios tiendan a ser la regla más que la excepción." (ob.cit., p. 178).

Refieren otras consecuencias como tener menos organización en el uso del tiempo (Feather, 1990) y reducción de los contactos sociales (Banks, Ullah y Warr, 1984; Jones, 1989). Entre los estudios clásicos efectuados en las décadas del 30 y 40, se describen efectos que no dejan de ser compartidos por los estudios de estos años, tales como:

El desempleo disminuye su conducta de asistencia a clubes y organizaciones voluntarias, el uso de la biblioteca pública y el hábito de leer. Su sentido del tiempo se desintegra, el no tener nada que hacer va asociado a la incapacidad de asistir puntualmente a las comidas u otros actos. 
La administración de sus recursos económicos, mucho más necesaria que antes, es progresivamente abandonada. Aunque las relaciones familiares continuaban en la forma habitual existía la evidencia de que sufrían más deterioro que otras relaciones y actividades (Jahoda, en Peiró y Prieto, 1996, p.227).

Esta autora advierte también de otros efectos como desesperanza, apatía y, principalmente, resignación.

Produce disminución de la vida social, de la cohesión familiar y de la confianza en sí mismo, al tiempo que aumenta la inseguridad social (Bakke, en Peiró y Prieto, 1996, 228).

Existencia de síntomas de ansiedad, nerviosismo, apatía, depresión y sentimientos de soledad y aislamiento en los desempleados (Trust, Ibid). Está relacionado con la inestabilidad emocional, pérdida general de ánimo, distorsión de la percepción del tiempo y aislamiento social. (Eisemberg y Lazersfeld, Ibid).

Ser alguien que no trabaja es un signo de "ciudadanía de segunda clase" y se asocia fuertemente con sentimientos de disminución de la dignidad personal. (Neff, 1972, p.186).

Una descripción detallada y patética de la experiencia por la que atraviesa el desempleado nos ofrece Forrester, que pinta el "terror frío" que acompaña al hecho de debatirse "entre las innumerables complicaciones, vejaciones y humillaciones de todo tipo que acompañan al desempleo" (ob. cit., p. 137).

En su obra es posible encontrar los matices de las consecuencias del desempleo de una manera que se aproxima mejor a nuestras necesidades descriptivas de la experiencia personal. Los efectos que destaca en cuanto a personalidad son los sentimientos de vergüenza y subordinación. Nos dice:

Vergüenza, ese sentimiento de ser indignos que conduce a la sumisión plena ... resignación mortificada (p. 14).

Esa vergüenza altera al individuo hasta la raíz, agota las energías, admite cualquier despojo... paraliza cualquier resistencia, impide... la situación (pp. 14-15).

Profana la identidad (p.55).

Marginación social (p.55). 
Pierden energías. Por eso son presa de un dolor subterráneo, efervescente, que provoca rabia y abatimiento a la vez (p.69).

Provoca desamparo, inseguridad y sentimientos de vergüenza (p. 137).

También se cuentan las opiniones y estudios de autores peruanos que ofrecen sus versiones bajo la idea general compartida de que "al desempleo generalmente se asocia la situación dramática de una familia hambrienta como consecuencia de la falta de trabajo del jefe de familia" (Verdera, 1983, p. 81). Cotler, en Cuadernos Laborales, y Manrique en su obra citada enfatizan en los derivados de violencia social y delincuencia. En un reciente congreso de psicología clínica, se presentaron tres ponencias que tocan el tema admitiendo la similitud general entre los "perfiles" de reacción aquí observados y los descritos en otros países. En lo pertinente a nuestro tema dicen:

Depresiones, sentimientos de angustia, ira, sentimientos de abandono y soledad (Ramirez., 1997).

Experiencia de pérdida ... La realidad de una experiencia sumamente dramática. (Tarazona y Maisch, 1997).

Su mundo se les acaba ... sienten que sus conocimientos) experiencia son pocos... sentimiento de imposibilidad de obtener empleo... actúan impulsados por la ansiedad ... falta de esperanza y fe en sí mismos. (Moore, 1997).

Como hemos podido observar, las consecuencias que refieren los autores señalan unas veces a toda la personalidad o a sectores amplios de la experiencia personal, y otras veces a sectores más restringidos o específicos de la experiencia. En ese sentido, un primer intento de agrupación puede ubicar las consecuencias en la relación presentada en el Cuadro 1. La relación presentada no pretende ser la síntesis de todas las consecuencias indicadas por los diferentes autores; pero sí estamos seguros de que refleja el contenido de la experiencia dramática que aqueja a quienes han sido privados de empleo y sobre lo cual los distintos autores pueden mostrar conformidad. A la vez, la relación se nos presenta sugestiva para estimular el trabajo de búsqueda y de comparación, pues nos puede ser, útil como marco de referencia al cual oponer los resultados de nuestra propia indagación. 


\section{CUADRO 1. CONSECUENCIAS PSICOLÓGICAS DEL DESEMPLEO}

Sectores Integrados de la personalidad:

- Menor bienestar psicológico.

- Marginación Social, reducción de los contactos sociales.

- Inseguridad social, desamparo, abandono.

- Inestabilidad emocional.

- $\quad$ Tendencia a la violencia social, a la delincuencia.

- Sumisión plena, sometimiento, subordinación, admite ser despojado.

- Impide enfrentar la situación. incapacidad para el afrontamiento.

- Profana la identidad.

- Dolor subterráneo que provoca rabia y abatimiento.

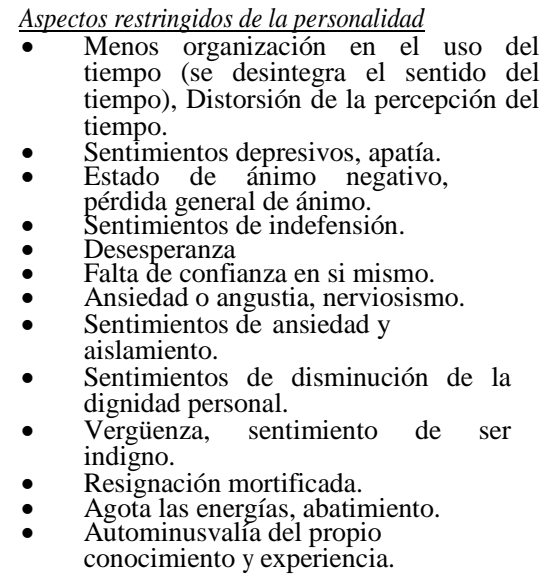

\section{EL PROBLEMA}

Hemos hecho una breve reseña de los distintos aspectos que interesan a la psicología en relación al desempleo, y entre ellos circunscribimos nuestro tema a la relación que se da entre la condición de desempleo y el impacto o efectos que produce en la experiencia personal de los desempleados. Hemos visto también que estos efectos han sido objeto de estudio en el exterior y con muestras de otros países; pero de nuestro medio sólo conocemos versiones emitidas a modo de opinión o comentario sin respaldo de investigación específica (en los últimos días nos hemos enterado de un estudio que se realiza en la Universidad de Lima aparentemente con el mismo propósito y distinta metodología). Nosotros nos preguntamos si los efectos descritos en bibliografía se repiten o son los mismos en nuestro medio, es decir, nos interesa conocer las particularidades que ocasiona el desempleo en la personalidad de nuestros trabajadores o, concretamente, entre los que sufren despido laboral en nuestra ciudad capital. 


\section{JUSTIFICACION}

El estudio parte de la necesidad de conocer la repercusión negativa del desempleo en la personalidad de nuestros trabajadores. Busca saber si la forma en que son afectados difiere o es semejante a lo que se observa en otros lugares. A la psicología y a la profesión interesan este deslinde porque, de registrarse diferencias, estaríamos abriendo el camino para definir posibles variables de personalidad que, por un lado permitan comprender la experiencia local del fenómeno y. por otro, replantear las modalidades y técnicas de asistencia psicológica. Siendo un fenómeno social, el desempleo tiene que adoptar particularidades según el medio donde ocurra y es conveniente que éstas se precisen en aras del acierto de la intervención psicológica

\section{OBJETIVOS}

1. Precisar la forma en que repercute el desempleo en la personalidad de los trabajadores de nuestro medio.

2. Detectar los aspectos de personalidad más sensibles al impacto negativo del desempleo.

Estos objetivos se ciñen a la observación de la personalidad de un número limitado de trabajadores de nuestra ciudad capital.

\section{TERMINOS BASICOS}

\section{Desempleo:}

El término adopta varias acepciones y clases. Nosotros nos referimos al desempleo abierto que, en la precisión de Verdera "comprende a las personas que habiendo trabajado ya no lo hacen (cesantes) y buscan activamente trabajo y a los aspirantes a trabajar, o sea. a los que buscan trabajo por primera vez" (ob. cit., p. 72). Y el añadido siguiente: 
"El desempleado abierto, también denominado declarado o activo, es un desocupado contra su voluntad y por este motivo es considerado en la PEA". (p. 73).

\section{Personalidad:}

Allport define la personalidad como "La organización dinámica dentro del individuo de aquellos sistemas psicofísicos que determinan sus ajustes únicos a su ambiente" (ob. cit., p. 65.). Pero también admite otras definiciones y una que cita como particularmente útil es la que la describe como "una sólida organización de las disposiciones y los sentimientos" (Ibid). Este sentido de rasgos estables y típicos es lo que centra la comprensión del término. Rasgos como los descritos en el listado del apartado 1.2., pero como características individuales, y esto son los que fundamentalmente detectan los tests de personalidad, que van a servir a nuestros propósitos.

\section{METODO}

Nuestro propósito de estudio apunta a los cambios que ocurren en la personalidad por influjo del desempleo; pero no los cambios transitorios y efervescentes que resultan de la reacción de alarma debida a la pérdida que acaba de sufrirse al momento del cese o despido, que generalmente pasan como reacciones temporales, sino a los rasgos estables y únicos que adquieren arraigo en el transcurso del tiempo, a lo que queda como efecto permanente y que se incorpora a la personalidad imprimiéndole un sello particular. Si se habla de personalidad se remite a lo estable en el comportamiento y no a lo pasajero. Por esta razón es que buscamos en el estudio a trabajadores que, por lo menos, tengan un año de cesados contra su voluntad y que buscan activamente colocación.

Este requerimiento se cumple a cabalidad entre los trabajadores despedidos de la Municipalidad de Lima que fueron cesados a mediados de 1996. Durante el año transcurrido de cese han enjuiciado al municipio y no cesan en sus protestas y movilizaciones reclamando su reposición. Ellos estaban organizados en varios sindicatos y nosotros establecimos contacto con el de empleados, 
SITRAMUN, que representaba a 1,080 despedidos, y con el permiso de su dirigencia asistíamos a su local para cumplir con nuestro propósito que fue suficientemente esclarecido. Una corta encuesta demográfica iniciaba la entrevista y disponía para alcanzar la atmósfera de evaluación, no siendo raros los que se abstenían de colaborar. Luego pasamos a las oficinas del municipio para cumplir igual tarea con el grupo de los empleados que continuaban trabajando y que aceptaban colaborar desde sus propios escritorios. De ese modo obtuvimos la muestra de comparación, basada en un sector homogéneo de población donde el desempleo sentaba la diferencia.

El encuentro de los rasgos estables y característicos de personalidad, exigía el empleo de un test de personalidad que sea reconocido como es el Psicodiagnóstico de Rorschach en su moderna variante de Exner. Como se sabe, esta prueba permite un análisis de respuestas que provee de puntajes, proporciones y constelaciones de símbolos que a su vez conducen a índices diagnósticos que describen la personalidad individual. Cada protocolo de prueba se sintetiza en un resumen estructural sobre el cual se registran los números que hacen las semejanzas y las diferencias una vez tamizados por el estadístico "t" de Student al nivel 0.05 de significación. Es el tipo de estudio que suele formularse con el Rorschach:

Estas son investigaciones en las cuales el Rorschach es aceptado como válido para propósitos específicos. Estos diseños ayudan a explorar diferencias relativas a comparación con grupos que han compartido una experiencia común. Estos son esencialmente estudios transversales y algunas veces han contribuido mucho al conocimiento y a la comprensión de los resultados Rorschach. En la mayoría de estas investigaciones, las variables Rorscharch generalmente se usan como variables dependientes (Exner 1995, p. 54, trad. de los autores).

El test de Rorschach es muy conocido entre los psicólogos, y nosotros lo usamos en su variante de Exner 94, que incorpora variaciones en el tratamiento de las respuestas. Sus procesos de administración, elaboración e interpretación los obviamos en esta oportunidad por ser cuestiones técnicas, y por haber sido expuestos en nuestros trabajos anteriores. Sólo añadiremos que la calificación 
de la prueba ha corrido a cargo de tres jueces para garantizar la confiabilidad del procedimiento.

En cuanto a las limitaciones del trabajo, debemos tener presente que: a) aprovechamos solamente la parte del Psicodiagnóstico de Rorschach que hace el análisis formal. lo que puede equivaler a trabajar con media prueba, dado que por el momento no efectuaremos el análisis del contenido de las respuestas que nos demandaría más tiempo del que disponemos, y b) el grupo del que hemos extraído nuestro material no es realmente la muestra sino sólo un grupo circunscrito que llama a prudencia en cuanto al alcance de los resultados.

\section{RESULTADOS}

Se comparan los dos grupos de protocolos Rorschach correspondientes a los empleados actuales, en número de veinte, y a los desempleados o despedidos, en número de treinta y cinco. Las características demográficas generales de ambos las muestra la Tabla 1 .

\section{TABLA 1. DATOS GENERALES DE LOS GRUPOS DE EMPLEADOS (20) Y DESEMPLEADOS (35) DE LA MUNICIPALIDAD DE LIMA.}

\begin{tabular}{|c|c|c|c|c|c|c|c|c|c|c|}
\hline \multirow[t]{2}{*}{ Condición } & \multicolumn{2}{|c|}{ Sexo } & \multicolumn{3}{|c|}{ Edad } & \multicolumn{2}{|c|}{ E. Civil } & \multicolumn{3}{|c|}{ Instrucción } \\
\hline & M & F & $18-25$ & $26-45$ & $46-60$ & Solt. & Cas. & Pri. & Sec. & Sup. \\
\hline Empleado & 10 & 10 & 2 & 9 & 9 & 13 & 7 & -- & 1 & 19 \\
\hline Desemp. & 21 & 14 & - & 13 & 22 & 12 & 23 & 3 & 9 & 23 \\
\hline $\mathrm{X}^{2}$ & \multicolumn{2}{|c|}{0.52} & & 5.84 & & \multicolumn{2}{|c|}{7.64} & \multicolumn{3}{|c|}{6.80} \\
\hline
\end{tabular}

El Chi cuadrado abona por la homogeneidad de los grupos en cuanto a sexo, edad e instrucción; no así en estado civil por el predominio de casados o convivientes entre los despedidos y de solteros entre los empleados, situación que debe tenerse presente para el momento de las interpretaciones.

De los resúmenes estructurales se obtienen 101 variables Rorschach de las cuales se calculan promedios y desviaciones estándar por grupo 
comparado, para fijar los valores " $t$ " que establecen las diferencias tal como aparecen en la Tabla 2, donde se señalan con asterisco las variables que han resultado con diferencia significativa que en total suman dieciséis. De éstas, predominan tres en los desempleados y las trece restantes en los empleados.

\section{TABLA 2. ANALISIS COMPARATIVO DE VARIABLES RORSCHACH EN EMPLEADOS Y DESEMPLEADOS DE LA MUNICIPALIDAD DE LIMA METROPOLITANA}

\begin{tabular}{|c|c|c|c|c|c|}
\hline \multirow{2}{*}{ VARIABLES } & \multicolumn{2}{|c|}{ Empleados (20) } & \multicolumn{2}{c|}{ Desempleados(35) } & \multirow{2}{*}{ "t" } \\
\cline { 2 - 5 } & Promedio & DS & Promedio & DS & \multirow{2}{*}{ (1.46* } \\
\hline D & 10.70 & 6.20 & 7.43 & 5.70 & $2.46^{*}$ \\
\hline Dd & 1.80 & 1.73 & 3.20 & 1.78 & $-2.83^{*}$ \\
\hline DQ+ & 3.10 & 2.45 & 4.66 & 2.45 & $-2.39^{*}$ \\
\hline Dqo & 15.25 & 8.16 & 10.80 & 3.69 & $2.78^{* *}$ \\
\hline Fqxo & 13.00 & 4.63 & 10.97 & 2.46 & $2.12^{*}$ \\
\hline FQxsin & 0.40 & 0.59 & 0.05 & 0.24 & $3.02^{* *}$ \\
\hline C Pura & 0.40 & 0.50 & 0.11 & 0.32 & $2.57^{*}$ \\
\hline C' & 0.20 & 0.41 & 0.74 & 1.17 & $-2.00^{*}$ \\
\hline FD & 0.65 & 0.59 & 0.34 & 0.54 & $1.97^{*}$ \\
\hline P & 6.20 & 1.47 & 4.60 & 1.63 & $3.62^{* *}$ \\
\hline Hx & 1.05 & 1.47 & 0.28 & 0.86 & $2.44^{*}$ \\
\hline SumH & 5.80 & 2.84 & 4.11 & 3.03 & $2.01 *$ \\
\hline Ls & 0.55 & 1.05 & 0.08 & 0.37 & $2.38^{*}$ \\
\hline DV1 & 0.45 & 0.68 & 0.05 & 0.24 & $3.10^{* *}$ \\
\hline AB & 0.30 & 0.57 & 0.06 & 0.24 & $2.22^{*}$ \\
\hline PER & 1040 & 1.54 & 0.54 & 0.95 & $2.56^{* *}$ \\
\hline
\end{tabular}

* Significativo al 0.05

** Significativo al 0.01 


\section{Análisis interpretativo}

\section{Variables diferenciales}

Veamos a continuación las designaciones y los significados generales y aislados de cada una de estas variables, comenzando por las que predominan entre los despedidos.

Dd Respuesta de pequeño detalle: Preferencia por lo pequeño, insignificante o sutil -Necesidad de mayor gasto de energía para procesar los estímulos- Reducción o alteración del contacto social -Restringe el campo de percepción y el sentido común como forma de evasión de las situaciones complejas- Incomodidad o inseguridad respecto de la propia capacidad para tomar decisiones.

DQ+ Calidad evolutiva de síntesis: Calidad sofisticada en el procesamiento de estímulos -Se aplica con dedicación y cuidado a la tareaGran necesidad de logro o afán de resarcimiento.

C' Respuestas de color acromático: Relacionada con aspectos depresivos, descenso del tono afectivo -Restricción de la expresión emocional con irritación interna y experiencia de incomodidad y tensión- El afecto debe ser contenido: un "morderse la lengua" psicológico, con emoción contenida que genera irritación -Desesperación con la que se convive y se admiten resignadamente Sentimientos de culpa o culpabilidad por algo transtornante que ya ha ocurrido. Propensión a enfermedades psicosomáticas.

Y seguidamente las designaciones y significados generales y aislados de las variables que predominan entre los empleados actuales.

D Respuestas de detalle: Expresión de percepción de lo inmediato, sencillo, práctico, como factor de buen sentido o sentido común Capacidad para el contacto social - Pensamiento de tipo práctico.

DQo Calidad evolutiva ordinaria: Predomina en trabajadores estables - 
Esfuerzo por procesar los estímulos en forma más modesta y conservadoraEvita la complejidad, opta por lo conocido y práctico.

FQxo Calidad formal ordinaria: Adecuación perceptiva, juzga como los demás, convencionalidad.

FQx sin Respuestas sin calidad formal: Falta de control cognitivo o racional en los estados ideacionales y reacciones emocionales. e pura Respuestas de color puro: Tendencia a descontrolarse, representan la impulsividad, las emociones no socializadas -Descarga emocional sin la mediación que se supone del pensamiento, carente de función lógicaIrreflexión, ira, accesos de cólera, agresividad.

FD Forma dimensión: Aptitud para tomar distancia y hacer una autoobservación objetiva de sí mismo; autobalance de lo positivo y negativo de sí.

P Respuesta popular: Capacidad para percibir lo obvio y convencional Capacidad para percibir y reaccionar como el común de la gente- Manejo de la realidad de forma convencional, concordante con la expectativa social.

Hx y Sum H: Contenido de experiencia humana y todos los contenidos humanos: Indicador de la capacidad para las relaciones interpersonales Interés por la gente (no necesariamente positivo).

LS Contenido de paisaje: Componente del índice de aislamiento Ideas o deseos de evadirse o alejarse de la realidad -Aislamiento y alejamiento afectivo.

DV1 Verbalización desviada de nivel 1: Desliz cognitivo relativamente moderado -Breve momento de desvarío cognitivo por empleo distorcionado del lenguaje o forma idiosincrática de expresión que disminuye la capacidad para comunicarse con claridad.

AB Contenido abstracto: Parte del índice de intelectualización -Táctica defensiva para neutralizar el efecto que produce la emoción, con riesgo a distorsión de la realidad.

PER Respuesta personal: Actitud defensiva tendiente a limpiar o justificar la auto imagen -Se apoya en la propia experiencia para sentirse resguardado del cuestionamiento de otros. 


\section{Relaciones entre variables}

Aparte de estos significados aislados, se cuenta con los que derivan de las relaciones entre las variables, como los siguientes:

- El aumento de Dd correlativa a una disminución de D se produce cuando concurren factores de estres con estados de ansiedad concomitantes donde el sentido común y el contacto con la realidad se debilitan. El sujeto se siente apabullado por los sucesos y se repliega

- La disminución de D, P, FQxo y el incremento de Dd refuerzan la tendencia de evitación de lo práctico, convencional. y obvio, reduciendo su accionar y complejizando las situaciones más de lo que son realmente.

- La disminución de Sum H junto con la de D, P, Y PER significan también disminución del contacto social, de las relaciones interpersonales y de la consideración por los demás, produciendo distanciamiento de las normas sociales y proclividad al comportamiento asocial.

- C' representa la cronicidad del problema traumático asociando la "constelación del niño quemado" o presencia permanente del problema, sumado a ello la falta de $\mathrm{P}$ tenemos nuevamente el retraimiento emocional y repliegue de lo social donde no se responde al mundo externo por temor a ser defraudados; se acompañan sentimientos de abandono y soledad, dudas sobre su capacidad para enfrentar problemas y la constante experiencia penosa e incomoda de tensión derivada de la inhibición de las emociones.

- C pura, Hx y FQx sin, que predominan en los empleados remiten a decaimiento en el control sobre los afectos y emociones, es decir, a espontaneidad o soltura emocional que no se permiten los despedidos.

- AB, Ls y FD, en el mismo sentido, son formas de defensa del yo que intervienen para mitigar el dolor de los encuentros desafortunados, que sirven a los empleados actuales y que escasean entre los despedidos. 
Las variables que permiten centrar toda esta información son el incremento de $\mathrm{Dd}$ y de $\mathrm{C}$ y la falta de $\mathrm{P}$ que reflejan la constricción dolorosa a que se halla sometida la personalidad. Los significados registrados por el Rorschach ofrecen los rasgos de personalidad característicos del grupo de despedidos que hemos evaluado.

\section{Agrupaciones por áreas de la personalidad}

En la esfera cognitiva: Aparece una reducción del campo de percepción y del sentido común, con apartamiento del modo corriente, usual o convencional de pensar y la opción por refugiarse en explicaciones menudas y relaciones inusitadas sobre asuntos pequeños o triviales. A esto acompaña recelo e inseguridad sobre la propia capacidad para tomar decisiones (ver gráfico 1).

\section{GRAFICO 1. COMPARACION DE PROMEDIOS DE VARIABLES COGNITIVAS RORSCHACH EN EMPLEADOS Y DESPEDIDOS}

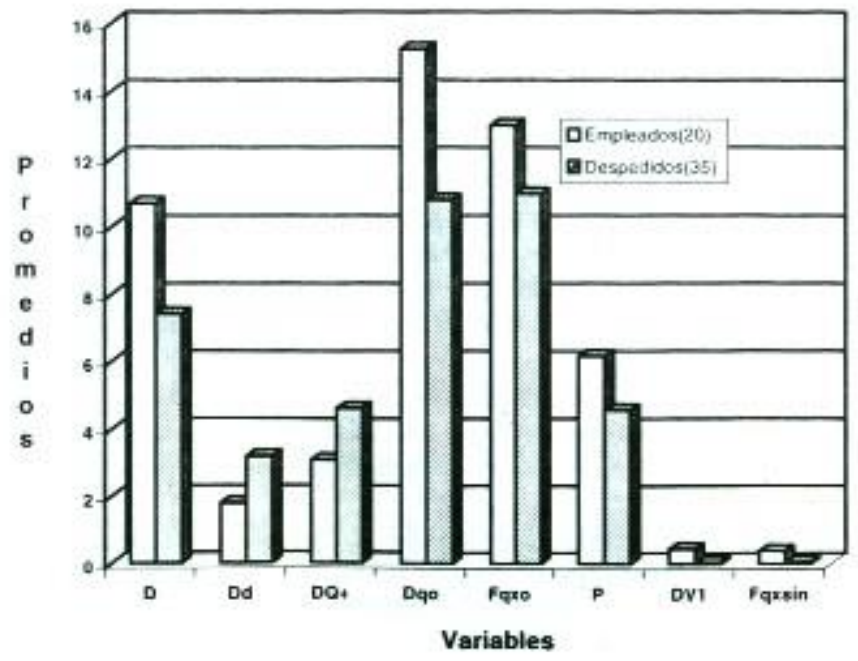


En el área emocional: Se vive una experiencia estresante con restricción de la expresión emocional, tensión, irritación y sentimientos culposos y de abandono social. (ver gráfico 2).

\section{GRAFICO 2. COMPARACION DE PROMEDIOS DE VARIABLES AFECTIVAS RORSCHACH DE EMPLEADOS Y DESPEDIDOS}

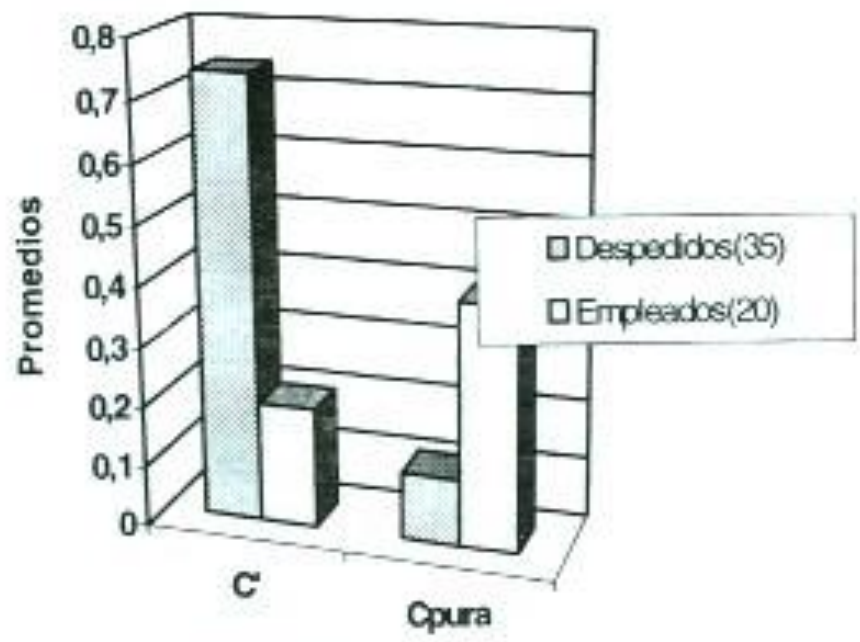

Variables

En el área interpersonal y de la autopercepción: La restricción alcanza también la esfera del contacto social con actitudes de repliegue y evitación. Se percibe, además, la ausencia de algunos mecanismos de defensa que sí amparan a los empleados actuales, en una situación que puede significar pérdida o abandono de los propios recursos de compensación o autodefensa, vale decir, debilitamiento y 
autoconstricción de la personalidad (ver gráfico 3).

\section{GRAFICO 3. COMPARACION DE PROMEDIOS DE VARIABLES RELACIONADAS A LA AUTOPERCEPCION DEL RORSCHACH EN EMPLEADOS Y DESPEDIDOS}

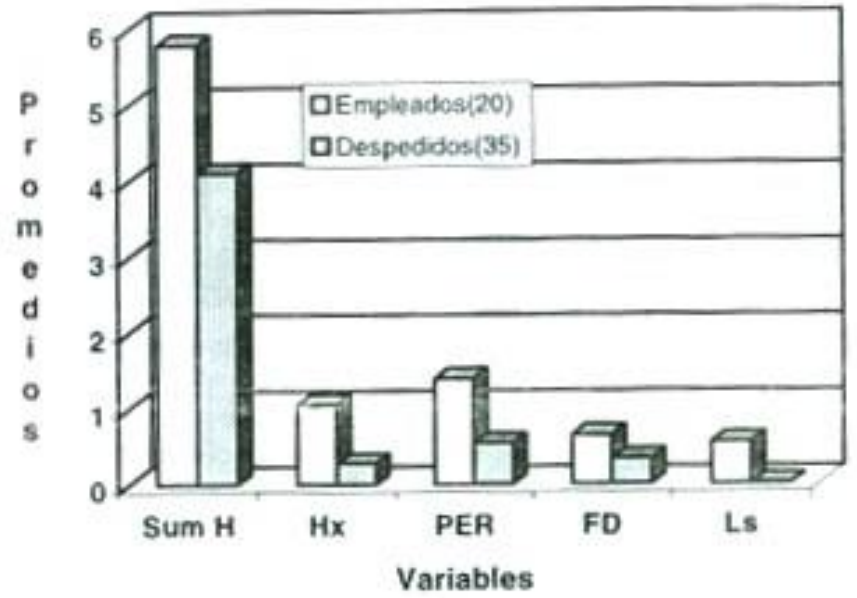

Entre esta síntesis y otros rasgos de detalle que acabamos de enumerar pueden encontrarse los rasgos que enlistamos en el punto 1.2 sobre las consecuencias o el impacto del desempleo en las personas; hay mucha similitud entre ambos conjuntos y no es exagerado si los hacemos equivalentes unos a otros, salvo en lo que concierne a dos rasgos de aquella relación y a dos de los aquí encontrados. Allí había la mención a profanación de la identidad y a distorsión en la percepción del tiempo que no ha sido posible reconocer en nuestra búsqueda. Aún cuando el rasgo de 
identidad mancillada es deducible del conjunto habido, parece carecer de argumento Rorschach.

Los dos rasgos aquí vistos pueden considerarse como el hallazgo de nuestro trabajo y son: la reducción del campo de percepción ( $\underline{\underline{D} d)}$ con igual reducción del sentido común (P $\downarrow, D \uparrow, D Q+\uparrow)$ y el enmarañamiento dentro de ese campo estrechado de información irrelevante y menuda. También debe sumarse como otra característica de nuestros despedidos su propensión a caer en enfermedades psicosomáticas $\left(\mathrm{C}^{\prime}\right)$, rasgo que tampoco se halla en el listado de bibliografía. Los dos rasgos que evaluamos como nuestro resultado [mal tienen importancia capital para los objetivos de asesoramiento y asistencia psicológica al despido.

\section{DISCUSION}

Los resultados que acabamos de ver deben tamizarse por las tres advertencias que hemos señalado y que conviene recordar ahora:

1) Nuestro trabajo evaluativo ha empleado solamente un sector de la prueba de Rorschach, el que corresponde a su análisis estructural, y por tanto no hemos agotado todas sus potencialidades diagnósticas; 2) Se ha evaluado a un grupo limitado de trabajadores y no a una muestra representativa de ellos, 3) Los ex trabajadores del estudio tienen un año de despedidos y en su mayoría son casados o convivientes, y se les ha comparado con un grupo de empleados actuales que en su mayoría son solteros, situación que incluye un factor de sesgo por responsabilidad familiar que, sin embargo, pensamos, no resta mucho al impacto que hemos descrito.

Ahora bien, teniendo en cuenta lo obtenido podemos decir que el cuadro general que agobia a la personalidad de los despedidos es una experiencia de exclusión o apartamiento social que es resultado obvio del despojo de que han sido objeto. Esta exclusión social lo induce a reaccionar con una restricción general del funcionamiento de la personalidad, expresada en un severo replegamiento cognitivo, emocional y social. La persona vive tensa $\mathrm{y}$ acorralada por sucesos que en rigor han sobrepasado gravemente su capacidad de control. El despido laboral es un estresor que violenta sin compasión la personalidad total infligiéndole desestabilización, constricción 
y un estado emocional de temor e indefensión. Los efectos sobre la personalidad son destructivos e incapacitantes y de tan vasta repercusión que más que enumerar o seleccionar los sectores que más afecta, que son todos, conviene decir que llega hasta alterar la capacidad de percepción y juicio reduciendo en el trabajador sus posibilidades de comprensión y ajuste a las situaciones vitales.

Estos resultados responden a los objetivos que nos habíamos fijado de ver la forma en que repercute el desempleo y señalar los aspectos de la personalidad que le son sensibles. Toda la personalidad es afectada y es por eso que confluyen los indicadores diagnósticos que los autores antes mencionados y nosotros mismos acompañamos al desempleo. Entre los resultados de unos estudios y de otros parece no haber discrepancias sino que se destacan más unos aspectos que otros y se van complementando en la necesidad de hacer tomar conciencia de los estragos del desempleo y del despido laboral. El sentido de complementariedad es el criterio que parece unificar el listado del punto 1.2 y la enumeración de nuestros resultados que guardan equivalencia hasta en lo que toca al distanciamiento de la normatividad social y sus derivados de violencia social. En cuanto a los cuatro rasgos que parecen no ser compartidos por ambos listados expresan más bien el aporte de cada uno a la mejor comprensión del tema. Lo más saltante del listado consultado que no aparece con nitidez en nuestra enumeración de rasgos se refería a la identidad profanada de la Forrester que no podemos dejar de aceptar tras reconocer el arrasamiento infligido por el despido. Nosotros hemos visto su señal en la insuficiencia de Hx y SumH, que son respuestas de contenido humano, pero la consideramos exigua para denotar la calidad de aspecto integrado de la identidad personal, y lo más probable es que ésta se vislumbre mejor en un análisis de contenido de las respuestas que seria deseable efectuar. Y los dos rasgos del hallazgo caen en ese cuadro como precisiones cognitivas complementarias a la descripción de la personalidad afectada.

Estos dos rasgos de la esfera cognitiva que hemos definido como restricción del campo de percepción y del sentido común y el refugio en fruslerías como opción para el tratamiento de la información, tienen la ventaja adicional de permitir una descripción más cercana a 
la experiencia íntima del despido en cuanto a cómo ve las cosas y cómo las entiende, situación que llama a reflexión sobre la índole del intercambio dialogal que puede exigir el trámite de asistencia psicológica a despedidos laborales, que de no ser advertida estaría dando luz verde a una fractura cognitiva profesionalmente imperdonable. El rasgo que expresa esa dedicación afanosa por la trivialidad menuda puede ser al mismo tiempo que efecto del repliegue una defensa compensatoria que hace suponer que se conserva la plenitud de la función intelectual. Es deducible la conveniencia de observar en la práctica las condiciones que imponen ambas restricciones en el curso de la entrevista y la comunicación.

Respecto a lo que se puede considerar como el hallazgo de nuestro trabajo, en primer lugar, no podemos asegurar que no se haya dicho o encontrado antes, dado que sólo hemos podido consultar una parte limitada de la extensa bibliografía sobre las consecuencias del desempleo. En segundo lugar, tampoco puede asegurarse que los rasgos de personalidad que hemos destacado ocurran solamente en los trabajadores evaluados o que, en general, sean características de nuestros trabajadores porque pueden ser detalles no cogidos en otras observaciones o estudios. Lo único que sí podemos afirmar, y en eso ha consistido nuestro trabajo, es que son rasgos destacados por el Psicodiagnóstico de Rorschach en un grupo de estudio integrado por desempleado s tras sufrir un año de su despido laboral. En buena cuenta, el punto de vista Rorschach de los efectos de la desocupación por despido.

\section{CONCLUSIONES}

1. El desempleo por despido laboral produce efectos destructivo s o incapacitantes en la personalidad de los integrantes del grupo de estudio.

2. Estos efectos negativos aquí encontrados son similares a los descritos por los autores consultados, pero el Psicodiagnóstico de Rorschach incide particularmente en los siguientes rasgos de personalidad: 


\section{Dora Frisancho / Anal Fairlie}

a) Restricción del campo perceptual y del sentido común, con mengua del modo convencional de pensar y refugio en elucubraciones menudas y triviales.

b) Mengua de los recursos de autodefensa psíquica.

c) Propensión a enfermedades psicosomáticas.

\section{BIBLIOGRAFIA}

Allport, G.W. (1961). Psicología de la personalidad. Bs. As.: Paidós.

Bernedo J, y otros (1990). El empleo en el Perú. Lima: ADEC-ATC.

Boloña B.C. (1993). Cambio de rumbo. Lima: SIL.

Cotler, J.: En Cuadernos laborales; ADEC-ATC, N 120, Feb - Mar 1997.

Chaeholiades, M. (1992). Economía internacional. Bogotá: Me Graw-HilL

Exner, J.E., Jr. (1994). El Rorschach: un sistema comprehensivo. Vol. I Fundamentos Básicos. Madrid: Psimática.

-(1995). Issues and Methods in Rorschach Research. New Jersey: LEA.

- y Sendín, C. (1995). Manual de interpretación del Rorschach. Madrid: Psimátiea.

Forrester, V. (1997). El horror económico. Bs. As.: FCE.

Manrique, N. (1997). La sociedad vitual y otros ensayos. Lima: PUC.

Mooree C., F., otros (1997). En Programa y resúmenes del Congreso Internacional de Psicología Clínica. Universidad de Lima.

Morris, Ch.G. (1992). Psicología. México: Prentice-Hall

Neft, W.S. (1972). El trabajo, el hombre y la sociedad. Bs. As.: Paidós.

Peiró, J. M. Prieto, F. (1996). Tratado de psicología del trabajo. Madrid: Síntesis.

Ramirez, J.C. (1996). En Programa y resúmenes del Congreso Internacional de Psicología Clínica. Universidad de Lima.

Rovira, F.M. (1983). El Rorschach: Nuevas aportaciones de J.E. Exner al Sistema Comprensivo. Madrid: Siglo Veintiuno.

Tarazona S. y Maisch M. (1997). En Programas y resúmenes del Congreso Internacional de Psicología Clínica; Universidad de Lima.

Verdera, F. (1983). El empleo en el Perú, un nuevo enfoque. Lima: IEP. 